\title{
The Late Heavy Bombardment in the Inner Solar System: \\ Is there any connection to Kuiper Belt Objects?
}

\author{
Christian Koeberl \\ Department of Geological Sciences, University of Vienna, \\ Althanstrasse 14, A-1090 Vienna, Austria
}

Proceedings of the Workshop on Kuiper-Belt Objects, Antofagasta, Chile (Earth, Moon and Planets) Submitted: April 27, 2003

\begin{abstract}
Data from lunar samples (Apollo, Luna, and lunar meteorites) indicate that the Moon was subjected to an intense period of bombardment around 3.85 billion year ago (Ga). Different interpretations exist, which either take this as the tail end of an intense but declining accretion period, or which consider a spike in the accretion rate at that time. The latter is the so-called Late Heavy Bombardment. Considering the enormous amount of matter that is required to accrete in the inner solar system at that time, and problems with deriving this mass from the asteroid belt, it is suggested that the Kuiper Belt objects could be a source for this bombardment spike, possibly linked to the late migration of Neptune outwards in the solar system.
\end{abstract}

\section{Introduction}

It is currently assumed that the Earth and other planets formed by accretion of smaller objects; i.e., impact processes (collisions) were of great importance in the early solar system. Late during the accretion of the Earth (some time after $4.5 \mathrm{Ga}$ ago), probably when the Earth had about $70 \%$ of its final mass, it was most probably impacted by a Mars-sized body, which is the prevailing hypothesis for the origin of the Moon (e.g., Canup and Righter, 2000). The consequences of such an impact event for the proto-Earth would have been severe and would have included almost complete re-melting of the Earth, loss of any primary atmosphere, and admixture of material from the impactor. The material remaining in orbit after accretion of the Moon would have continued to impact onto the Earth (and the Moon) for millions of years or more. Core formation was coeval with the accretion and the core of the Mars-sized impact is likely to have merged with the core of the proto-Earth almost instantaneously. Due to later geological activity, no record of this very early bombardment remains on the surface of the Earth.

On the other hand, numerous data obtained from Apollo rocks that indicate that the Moon was subjected to intense post-accretionary bombardment between about 4.5 and 3.9 billion years ago (e.g., Wilhelms, 1987). In addition, some lunar data indicate that a short and intense late heavy bombardment (LHB) period occurred around $3.9 \pm 0.1$ Ga ago (e.g., Tera et al., 1974; Ryder, 1990); however, these data are disputed by some researchers (e.g., Baldwin, 1974; Hartmann, 1975). In the present 
contribution I am briefly reviewing the evidence for a LHB in the inner solar system (following Ryder et al., 2000) and discuss possible sources of the impacting objects, speculating that there could be a connection to Kuiper-Belt objects.

\section{Evidence for the Late Heavy Bombardment}

The accretion of the Earth appears to have been completed about 50 to $100 \mathrm{Ma}$ after the initial formation of the solar nebula, defining the beginning of the so-called Hadean Eon. Whereas there is almost no evidence of terrestrial witnesses to the Hadean Eon, the pre-Nectarian and Nectarian periods cover this time interval on the Moon. Soon after the formation of the Moon, the highlands crust formed. The morphology of the highlands of the Moon reflects almost exclusively a history of numerous impacts that occurred prior to the extrusion of the volcanic flows that form the visible mare plains (e.g., Wilhelms, 1987). Geochronological studies of brecciated highlands samples showed that impact-related thermal events concentrated at $\sim 3.8$ to $3.9 \mathrm{Ga}$. These ages have been taken to represent either the tail end of a heavy but declining bombardment (e.g., Hartmann 1975, Hartmann et al., 2000; Baldwin, 1974, Wilhelms, 1987) or a sharp and cataclysmic increase in bombardment for that short period (e.g., Ryder 1990; Kring and Cohen, 2002). These different interpretations are also illustrated in Fig. 1.

The stratigraphy of the highlands has been divided on the basis of basin formation and ejecta into pre-Nectarian System, Nectarian System, and Lower Imbrian Series. These are separated by the bottom of the Nectaris basin deposits, the bottom of the Imbrium basin deposits, and the top of the debris blanket of the Orientale basin, respectively (e.g., Wilhelms 1987). Several basins were produced during the Nectarian, including Serenitatis and Crisium, from whose ejecta we have samples (Apollo 17 and Luna 20). The Schrödinger basin is Lower Imbrian, as are several large craters including some almost $200 \mathrm{~km}$ in diameter. The oldest mare deposits are in the Upper Imbrian Series, whose uppermost boundary is defined in terms of crater degradation in the absence of any globally useful stratigraphicdatum horizons comparable to basin ejecta. The dating of these boundaries as well as of other basins within the stratigraphic units is what defines the chronology of lunar bombardment and the flux over the main period of interest here.

The best way to date an impact is from a clast-free or clast-poor impact melt, and Ryder (1990) discussed that there is a lack of impact melts in the sample collections that are older than $\sim 3.92$ to 3.95 $\mathrm{Ga}$; this is unlikely to be the result of resetting of all older ages, given the difficulties of such resetting. Thus that lack can be taken as evidence that there was little impacting prior to that time. Here is not the place to review the arguments for the ages of the large impact basins (Mare) on the Moon; the reader is referred to Ryder et al. (2000) for details. It just needs to be noted that all large impact basins have formation ages of about 3.8 to $4 \mathrm{Ga}$ (the magma that filled in these impact basins to form the Mare is of course younger, to about $3 \mathrm{Ga}$ ). For example, the most likely ages are: for the Imbrium Basin, $3.85 \pm$ $0.02 \mathrm{Ga}$; Nectaris, $\sim 3.90 \mathrm{Ga}$; Serenitatis, $3.893 \pm 0.009 \mathrm{Ga}$; and Crisium also $\sim 3.89 \mathrm{Ga}$, with several 
other basins, e.g., Herzsprung and Humorum, forming after Nectaris; thus, there was considerable bombardment of the Moon in the 60 million years between $3.90 \mathrm{Ga}$ and $\sim 3.84 \mathrm{Ga}$. This conclusion was supported by recent work of Cohen et al. (2000) and Kring and Cohen (2002), who showed that ages of impact melts from the lunar meteorites, which constitute random collections of lunar surface material, also cluster in the same age bracket. This period of bombardment terminated at $3.85 \mathrm{Ga}$ with the near simultaneous creation of the Imbrium, Schrödinger, and Orientale basins. Though the last two might have been as late as $3.80 \mathrm{Ga}$, this seems unlikely given their crater populations being almost as high as those on the Imbrium ejecta.

The data summarized by Ryder et al. (2000) indicate a massive decline in the flux of bombardment on the Moon over a short period of time. The cratering on top of the Nectaris ejecta (3.90 $\mathrm{Ga}$ ) is a factor of 4 higher than that on Imbrium ejecta, which is in turn a factor of 2 to 4 times that on the oldest mare plains $(\leq 3.80 \mathrm{Ga})$. Thus, during the period 3.90 to about $3.85 \mathrm{Ga}$ the flux was about 1000 times greater than it is now, and a few hundred times heavier in the subsequent 50 million years. It is possible that the decline took place over only the first 10 of that 50 million years, such that by 3.84 or $3.83 \mathrm{Ga}$ the flux was almost as low as that of the present day.

An argument can also be made regarding the masses of basin-forming projectiles on the Moon. Ryder $(2001,2002)$ gives a detailed discussion of this topic, which I will try to summarize here. The masses of the Imbrium and Orientale projectiles, for instance, have been estimated at between $8 \times 10^{20}$ to $2 \times 10^{21} \mathrm{~g}$ and $4 \times 10^{20}$ to $1.5 \times 10^{21} \mathrm{~g}$, respectively. Thus, the mass of the about 15 Nectarian and Early Imbrian basin-forming projectiles would be on the order of $10^{21}$ to $10^{22} \mathrm{~g}$; considering that the ages of these basins are within about 80 million years (see Fig. 1) leads to a lower limit of the accretion rate on the Moon about $1.2 \times 10^{13} \mathrm{~g} /$ year. This is about one to two orders of magnitude above the smooth decline curve of the lunar accretion, and three orders of magnitude above a back-extrapolated current accretion rate. If we assume a much longer age spread for these basins (Nectaris age at $4.12 \mathrm{Ga}$ ), the average mass accretion rate of at least $3 \times 10^{12} \mathrm{~g} /$ year for $300 \mathrm{Ma}$ is still about an order of magnitude larger than the back-extrapolated curve (solid line in Fig. 1). If one would assume that the mass accretion in the first case is the tail-end of the early lunar accretion and not a spike, then one arrives at the dotted curve in Fig. 1, which is unreasonable because it would lead accreting the Moon not at about $4.5 \mathrm{Ga}$ but at about 4.1 Ga, clearly at odds with all we know about the Moon. Thus, I follow Ryder (1990, 2001, 2002), Ryder et al. (2000), and Kring and Cohen (2002) in accepting the evidence for a cataclysmic LHB in the inner solar system.

\section{State of the Earth before $3.8 \mathrm{Ga}$}

Early in its history, the Earth must have been subjected to a significantly larger number of impact events than the Moon, as it has a larger diameter and a much larger gravitational cross section than the Moon. Thus, if a LHB occurred on the Moon, the Earth must have been subjected to an impact flux at 
least as intense. The consequences for the Earth must have been devastating. There is some evidence that the Earth's upper mantle had already undergone some differentiation at the time of formation of the oldest rocks preserved on the Earth's surface (e.g., Harper and Jacobsen, 1992; McCulloch and Bennett, 1993; Bowring and Housh, 1995; Boyet et al., 2003; but see also Papanastassiou et al., 2003); it was suggested that the absence of any rocks older than about $3.9 \mathrm{Ga}$ is the result of LHB, during which impact-induced mixing recycled early crustal fragments back into the upper mantle (Koeberl et al., 2000).

Very few rocks on Earth with ages approaching 3.9 Ga have been found; some rare older detrital zircon grains up to almost $4.3 \mathrm{Ga}$ are known. It is likely that the Moon-forming impact led to a large-scale melting of the Earth and the existence of an early magma ocean. Mantle temperatures in the Hadean were probably much higher than today as a result of a higher heat flow (about half of all heat produced by ${ }^{235} \mathrm{U}$ decay to ${ }^{207} \mathrm{~Pb}$ was released during the Hadean, adding several hundred degrees to the internal temperature of the Earth) and thermal energy released during the impact of late accretionary bodies (e.g., Davies, 1985). The nature and amount of the earliest crust on Earth has been debated, but comparison with other planets suggests that the earliest crust on Earth was basaltic (e.g., Taylor, 1989, Arndt and Chauvel, 1991). Morphological, mineralogical, and geochemical characteristics of the 4.2-4.3 Ga zircons indicate a composite granitoid source of continental provenance for these zircons. Thus, there is evidence for at least minor amounts of felsic igneous rocks in the Hadean, which may have formed in small amounts from remelting of basaltic crust that sank back into the mantle (e.g., Taylor, 1989). Thus, the Hadean Earth was most probably characterized by a thick basaltic crust, covered by an ocean, with little dry land and minor amounts of felsic rocks (granitoids). Any sedimentological record, which would host information specific to surface environments such as the rate and violence of meteorite impact and the presence of life, has been lost from Hadean times.

Samples of some of the oldest rocks on Earth, from Isua, Greenland, were analyzed by Koeberl et al. (2000) for their chemical composition, including the platinum group element (PGE) abundances, in an attempt to find a possible extraterrestrial component, similar to what is observed in some ejecta layers (e.g., Koeberl, 1998). Unfortunately, the results are ambiguous, and no clear meteoritic signature was found. In addition, petrographical studies of zircons extracted from these rocks failed to show evidence for shock metamorphism. Zircon is one of the most resistant minerals and would be expected to impact-related shock features if it was subjected to any impact events in the past. There are several reasons for not obtaining any evidence for a LHB on Earth from these samples. First, the number of samples may have been small. Second, very large impact events lead to the formation of large amount of impact melt, which may have destroyed any direct (shock) evidence. Also, while the Isua rocks are usually considered to be about $3.85 \mathrm{Ga}$ (e.g., Mojzsis et al., 1996), it has been argued that while the zircons that were dated are indeed $>3.8 \mathrm{Ga}$ old, the rocks may only be about $3.65 \mathrm{Ga}$ old (e.g., Moorbath and Kamber, 1998). In this case, the LHB would have long ceased and no direct evidence for an extraterrestrial component could be obtained. Indeed, the data and arguments of Mojzsis et al. (1996), 
who used carbon isotopic compositions of graphite in apatites from these Isua rocks to suggest that life could have evolved as early as $3.85 \mathrm{Ga}$, have been shown to be erroneous (e.g., van Zuilen et al., 2002; Fedo and Whitehouse, 2002). At any rate, the influence of impact events on early life must have been severe (e.g., Sleep et al., 1989; Zahnle and Sleep, 1997).

The only evidence for a LHB on the Earth is coming from recent tungsten (W) isotopic studies by Schoenberg et al. (2002), who found $\mathrm{W}$ anomalies in ca. 3.85 Ga metasedimentary rocks from Greenland, which at this time are difficult to explain by anything but an extraterrestrial component.

\section{Origin of the Late Heavy Bombardment}

Thus, while there is only limited evidence for a LHB on the Earth, the evidence from the Moon, which preserves the early impact history of the solar system so much better than the Earth, is convincing. Ryder (2002) showed that, if the masses of the impactors that formed the large basins at $3.8-3.9 \mathrm{Ga}$ were part of a tail of a declining impact flux, as early as $4.1 \mathrm{Ga}$ this flux would have reached masses equivalent to the mass of the moon. Thus, it is impossible to simple extrapolate the impact flux derived from the basin-forming impactors back in time. In turn, this provides further evidence for the existence of a spike in the impact flux (i.e., a LHB). Further evidence for a LHB has been found from Mars.

Therefore, it seems quite likely that a LHB really did happen in the inner solar. This brings on the question regarding the source of the impactors. It needs to be emphasized that the spike in the impact flux in the inner solar system could have been relatively steep, i.e., it could be that the time window was less than $100 \mathrm{Myr}$, because the current limits of our knowledge of the lunar impact ages are given by the precision of the geochronologic methods. The spike could have had a halflife of only 20 or $30 \mathrm{Myr}$. In addition, a supply of fairly large bodies is necessary. The impactors that formed the large lunar basins had to be several tens of kilometers in size (maybe up to $50 \mathrm{~km}$ diameter), and there must have been many of them. This makes a short-time disturbance of the asteroid belt a rather unlikely source. Recent studies in celestial mechanics led to the proposal of a possible mechanism that could plausibly supply a short-time spike in an otherwise steady or decreasing background flux of impactors (Zappalà et al., 1998); however, such sources need to be quantified. An investigation by Morbidelli et al. (2000) indicated that asteroids from the main belt are a rather unlikely source of impactors for the LHB because it is difficult to envision a mechanism that would deliver a short-time flux increase into the inner solar system.

Levinson et al. (2001) suggested that Uranus and Neptune did not form at the same time as the terrestrial planets and Jupiter and Saturn, but that their formation was delayed until about $3.9 \mathrm{Ga}$, and once these planets started accreting, which would have happened very rapidly (with 10 to $20 \mathrm{Myr}$ ), abundant planetesimals would be scattered throughout the solar system, and be responsible for the LHB. The possibility that Kuiper-Belt objects (KBOs) are somehow relevant for the LHB is an intriguing one. The mass flux into the inner solar system at the time of the LHB must have been significant. Values on 
ranging from about $6 \times 10^{21}$ to $10^{23} \mathrm{~g}$ of material accreted each by the Moon, Mars, and Earth have been calculated (e.g., Ryder, 2001, 2002; Levinson et al., 2001). It can be assumed that only a fraction of the objects entering the inner solar system would be accreted by the planets and the Moon; thus, the total amount of matter injected into the inner solar system could amount to a sizeable fraction of the total mass of the asteroid belt. There is no conceivable mechanism to destabilize several percent of the mass of the asteroid belt. In contrast, the mass of objects in the paleo-Kuiper Belt was several orders of magnitude larger than the mess of the asteroid belt. Destabilization of these objects would provide a much more plausible source of projectiles for the LHB.

According to Gomez (2003), Uranus and Neptune formed much closer to the sun than where they are now. Most planetesimals would be swept up by these protoplanets, which were slowly migrating outwards. It is at least qualitatively conceivable (A. Morbidelli, pers. comm., 2003) that within a few hundred million years after formation of Neptune it had slowly migrated into a planetesimal-rich zone, whereupon it started to accrete more mass and rapidly migrated outwards towards $40 \mathrm{AU}$, in the process scattering KBOs all over the solar system, leading to the LHB in the inner solar system and to the accumulation of the "hot" KBOs in the outer solar system. Open questions include the currently not well known timescale for the formation of Neptune (and Uranus), and if it is possible to distinguish from the remnants of the LHB on Earth and the Moon if the projectiles were of closer in composition to asteroids or to KBOs (which includes the problem how well known is the composition of $\mathrm{KBOs}$ - the proportion of rock to ice (+organics) could be 1:1, but the composition of the rocky part is not well known either, although it is assumed to be close to primitive solar matter). In summary, if we accept the evidence for a LHB (which in my opinion is fairly strong), then a source for the projectiles is necessary, and inner solar system sources appear not massive enough or are implausible for dynamical reasons. Qualitatively the KBOs could be a source, but the exact timescales and the dynamic conditions still need to be explored.

Acknowledgments: I would like to acknowledge my late colleague and friend Graham Ryder (19492002) for many interesting discussions and ideas over the years. I thank A. Morbidelli for motivating discussion during the Antofagasta workshop. This work is supported by the Austrian Science Foundation, grant Y58-GEO.

\section{References}

Arndt N., and Chauvel C. (1991) Crust of the Hadean Earth. Bull. geol. Soc. Denmark 39, 145-151.

Baldwin R.B. (1974) Was there a "Terminal Lunar Cataclysm" 3.9-4.0x109 years ago? Icarus 23, 157166.

Bowring S.A., and Housh, T. (1995) The Earth's early evolution. Science 269, 1535-1540. 
Boyet M., Rosing M., Blichert-Toft J., Storey M., and Albarede F. (2003) ${ }^{142} \mathrm{Nd}$ evidence for early earth differentiation [abs.]. Lunar Planet. Sci. 34, abs. \#1945 (CD-ROM).

Canup R., and Righter, K., eds. (2000) Origin of the Earth and Moon. Tucson, University of Arizona Press.

Cohen B.A., Swindle T.D. and Kring D.A. (2000) Support for the lunar cataclysm hypothesis from lunar meteorite melt ages. Science 290, 1754-1756.

Davies G.F. (1985) Heat deposition and retention in a sold planet growing by impacts. Icarus 63, 45-68.

Deutsch A. and Schärer U. (1994) Dating terrestrial impact events. Meteoritics 29, 301-322.

Fedo, C.M, and Whitehouse, M.J. (2002) Metasomatic origin of quartz-pyroxene rock, Akilia, Greenland, and implications for the Earth's earliest life. Science 296, 1458-1462.

Gomez R. (2003) Presentation at KBO Workshop, Antofagasta, Chile, March 2003.

Harper C.L., and Jacobsen S.B. (1992) Evidence from coupled ${ }^{147} \mathrm{Sm}_{-}{ }^{143} \mathrm{Nd}$ and ${ }^{146} \mathrm{Sm}-{ }^{142} \mathrm{Nd}$ systematics for very early (4.5-Gyr) differentiation of the Earth's mantle. Nature 360, 728-732.

Hartmann W.K. (1975) Lunar "cataclysm": A misconception? Icarus 24, 181-187.

Hartmann W.K., Ryder G., Dones L., and Grinspoon D. H. (2000) The time-dependent intense bombardment of the primordial Earth-Moon system. In: Origin of the Earth and Moon (Eds. R. Canup and K. Righter), University of Arizona Press, p. 493-512.

Koeberl C. (1998) Identification of meteoritical components in impactites. In: Meteorites: Flux with Time and Impact Effects (Grady, M.M., Hutchison, R., McCall, G.J.H., and Rothery, D.A., eds.), Geological Society of London, Special Publication 140, pp. 133-152.

Koeberl, C., Reimold, W.U., McDonald, I., and Rosing, M. (2000) Search for petrographical and geochemical evidence for the late heavy bombardment on Earth in Early Archean rocks from Isua, Greenland. In: Impacts and the Early Earth (Eds. I. Gilmour and C. Koeberl), Lecture Notes in Earth Sciences 91, Springer Verlag, Heidelberg, p. 73-97.

Kring D.A., and Cohen B.A. (2002) Cataclysmic bombardment throughout the inner solar system 3.94.0 Ga. Journal of Geophysical Research 107(2), 4-1 - 4-5.

Levinson H.F., Dones L., Chapman C.R., Stern S.A., Duncan M.J., and Zahnle K. (2001) Could the lunar "Late Heavy Bombardment" have been triggered by the formation of Uranus and Neptune? Icarus 151, 286-306.

McCulloch M.T., and Bennett V.C. (1993) Evolution of the early Earth: Constraints from ${ }^{143} \mathrm{Nd}-{ }^{142} \mathrm{Nd}$ isotopic systematics. Lithos 30, 237-255.

Moorbath S., and Kamber B.S. (1998) A reassessment of the timing of early Archaean crustal evolution in West Greenland. Geology of Greenland Survey Bulletin 180, 88-93.

Morbidelli A., Petit J-M., Gladman B., and Chambers. J. (2001) A plausible cause of the late heavy bombardment. Meteoritics and Planetary Science 36, 371-380.

Mojzsis S.J., Arrhenius G., McKeegan K.D., Harrison T.M., Nutman A.P., and Friend C.R.L. (1996) Evidence for life on Earth before 3,800 million years ago. Nature 385, 55-59. 
Ryder G. (1990) Lunar samples, lunar accretion, and the early bombardment history of the Moon. EOS Trans. Am. Geophys. Un. 71, 313-323.

Ryder G. (1994) Coincidence in time of the Imbrium basin impact and Apollo 15 volcanic flows: The case for impact-induced melting. In Large Meteorite Impacts and Planetary Evolution (B.O. Dressler, R.A.F. Grieve, and V.L. Sharpton, eds.). Geol. Soc. Am. Spec. Paper 293, 11-18.

Ryder G. (2001) Mass flux during the ancient lunar bombardment: The cataclysm [abs.]. Lunar Planet. Sci. 32, abs. No. 1326 (CD-ROM).

Ryder G. (2002) Mass flux in the ancient Earth-Moon system and benign implications for the origin of life on Earth. J. Geophys. Res. 107, doi 10.1029/2001JE001583, pp. 6-1 - 6-14.

Ryder, G., Koeberl, C., and Mojzsis, S.J. (2000) Heavy bombardment on the Earth 3.85 Ga: The search for petrographic and geochemical evidence. In: Origin of the Earth and Moon (Eds. R. Canup and K. Righter), University of Arizona Press, p. 475-492.

Schoenberg R., Kamber B.S., Kollerson K.D. and Moorbath S. (2002) Tungsten isotope evidence from $\sim 3.8$-Gyr metamorphosed sediments for early meteorite bombardment of the earth. Nature 418, 403-405.

Sleep N.H., Zahnle K.J., Kasting J.F., and Morowitz H.J. (1989) Annihilation of ecosystems by large asteroid impacts on the early Earth. Nature 342, 139-142.

Taylor S.R. (1989) Growth of planetary crusts. Tectonophysics 161, 147-156.

Tera F., Papanastassiou D.A., Wasserburg G.J. (1974) Isotopic evidence for a terminal lunar cataclysm. Earth Planet. Sci. Lett. 22, 1-21.

van Zuilen, M.A., Lepland A., and Arrhenius G. (2002) Reassessing the evidence for the earliest traces of life. Nature 418, 627-630.

Wilhelms D.E. (1987) The geologic history of the Moon. U.S. Geol. Surv. Prof. Pap. 1348, 302 pp.

Zahnle K.J. and Sleep N.H. (1997) Impacts and the early evolution of life, In Comets and the origin and evolution of life (Thomas P.J., Chyba C.F., and McKay C.P., eds.), pp. 175-208. SpringerVerlag, New York.

Zappalà V., Cellino A., Gladman B.J., Manley S., and Migliorini F. (1998) Asteroid showers on Earth after family breakup events. Icarus 134, 176-179. 


\section{Figure Captions}

Fig. 1. This diagram shows the various interpretations of the mass flux (accretion rate) on the Moon, modified after Ryder $(2001,2002)$. Triangles mark data from lunar sample studies, and the formation of the lunar highlands. Ages of a few major impact basins are indicated. The solid line is the presentday background flux extrapolated back in time towards the origin of the solar system; the dashed line is the elevated flux after Hartmann (e.g., Hartmann et al., 2000), and the curve marked LHB indicates the spike in the accretion rate that is suggested to be the Late Heavy Bombardment. The dotted line indicates an accretion curve that includes the masses of the basin-forming projectiles; this curve is unlikely because it leads to the accretion of the Moon at 4.1 instead of 4.4-4.5 Ga (indicated by the gray band $=$ the age of the Moon obtained from isotopic constraints).

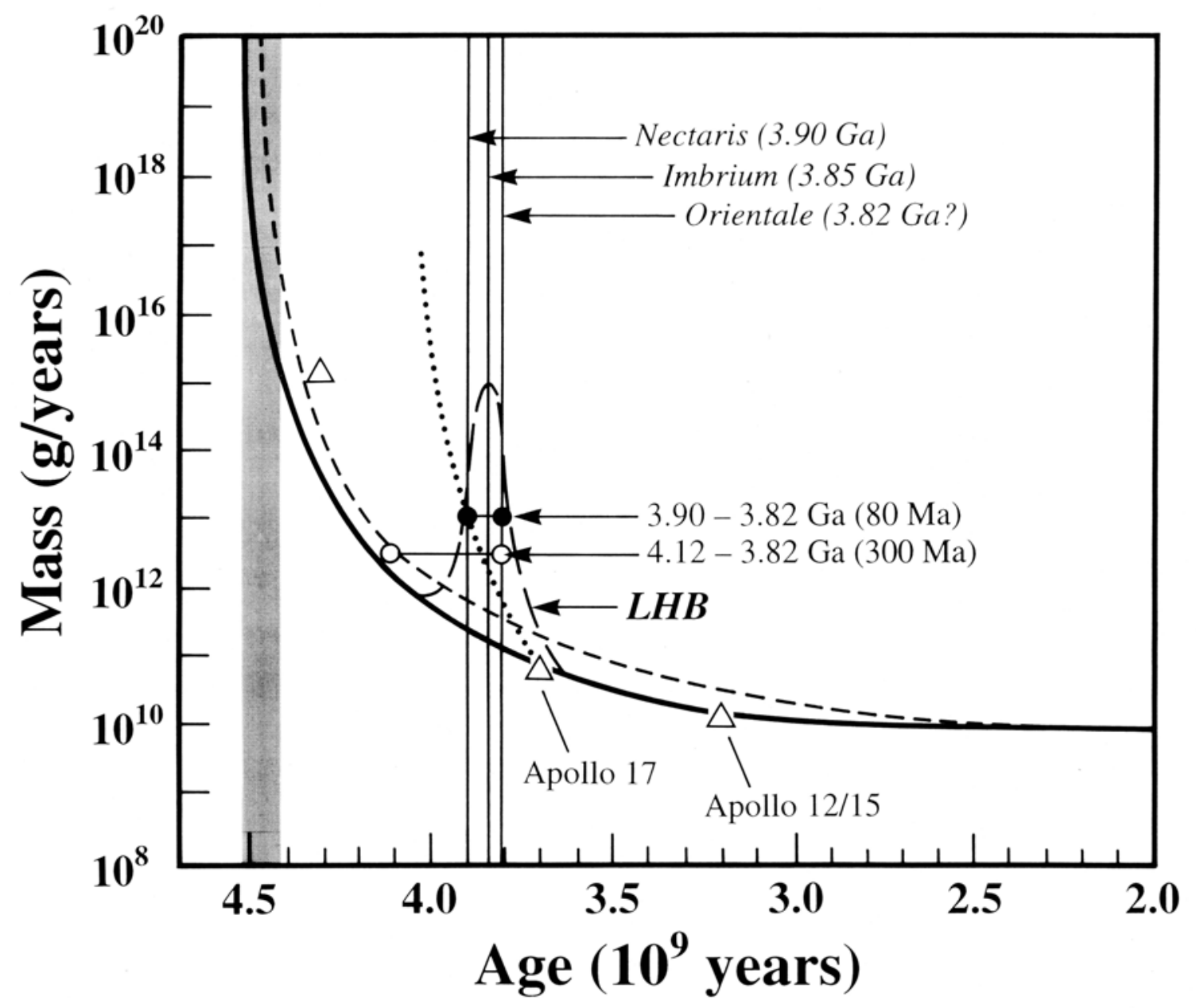

